\title{
6G TECHNOLOGY HEALTHCARE MANAGEMENT
}

\author{
RAKHI SETH $^{1}$, SUYASH AGRAWAL ${ }^{2}$ \& MEGHA SETH ${ }^{3}$ \\ ${ }^{I}$ Assistant Professor, National institute of technology, Raipur (CG), India \\ ${ }^{2,3}$ Assistant Professor, Bharat Institute of Engineering and Technology, Hyderabad, India
}

\begin{abstract}
The present usage of $5 G$ innovation has pushed the scholarly network to consider what is straightaway. To appropriately respond to this inquiry, we need to make sense of which will be the necessities later on. This paper manages the responses to those inquiries for the wellbeing vertical of $6 G$. The developed and the maturing of populace overall make the present social insurance frameworks impractical later on. Remote wellbeing must be executed to leave all residents alone followed and oversaw in the wellbeing procedure of their life by seeking after a financially practical route for the network. $6 G$ is imagined to be an innovation which won't just make the remote social insurance valid, however it likewise will permit the Internet of Bio-Nano-Things, leaving the human body alone piece of the "Net". Exceptionally low complex wearable/implantable gadgets will be a piece of our regular day to day existence, which can recuperate data about our wellbeing and way of life from each item we collaborate with, from a jug of water to a shrewd medication.

KEYWORDS: 6G, Future Health Care Services, Body Area Network, In-Body Communications, Human Bond Communications, Internet of Medical Things
\end{abstract}

Received: Jun 09, 2020; Accepted: Jun 29, 2020; Published: Aug 13, 2020; Paper Id.: IJMPERDJUN2020759

\section{INTRODUCTION}

The expanding number of incessant patients and the maturing of populace make the counteraction an obligatory necessity. Counteraction is characterized as an intend to keep a decent way of life (physical movement, diet, occasional wellbeing controls, and so on.), yet additionally as an intend to stay away from the intensifying of interminable conditions. One of the goals of the human services framework is to effectively add to the prosperity of residents. In this unique situation, characterizing progressed ICT answers for preventive consideration, wellbeing status observing and wellbeing and prosperity training by joining the data originating from natural, wearable and even in-body sensors is critical. It empowers the making of a system of continuous refreshed patients' clinical data, supporting the human services experts in dynamic. Future investments in research and innovation for health [1] will essentially focus to: I) solid residents in a quickly evolving society; ii) compelling wellbeing administrations to handle and lessen the weight of ailments; iii) improved access to imaginative, economical and great medicinal services. The advancement of more beneficial practices and ways of life and the arrangement of proficient ailments the executives including convenient moderate and available excellent medicinal services administrations depend on a superior comprehension of wellbeing and maladies, inventive methodological and mechanical administration arrangements, new economical methodologies for a high open computerized transformation and personalized and integrated needs-driven healthcare services.

The goal of this paper is to give a multidisciplinary diagram of how $6 \mathrm{G}$ innovation can change the future remote human services by thinking about various angles (mechanical, moral and administrative, sociological, and 
so forth.). $6 \mathrm{G}$ is viewed as a full and inescapable network texture which permits to get many/all the required data from people (sound and not), from how much water he/she has flushed in a day (for example from sensors on the container, or somewhere in the vicinity) to the initiation of on request wellbeing registration (for example by initiating sensors onto/into the body).

The paper is composed as follows: Section I gives an outline of the principle patterns of future research ventures for wellbeing; in Section II the new client needs are introduced by giving a few measurements on constant infections and maturing, while Section III reports the primary highlights of things to come remote social insurance administrations. A short review of the key components of $6 \mathrm{G}$ is given in Section IV to present how $6 \mathrm{G}$ innovation can change the social insurance framework. Area $\mathrm{V}$ centers on a portion of those $6 \mathrm{G}$ correspondence innovations that are required to be the future social insurance administrations empowering agents. Difficulties on security and protection, specialized, moral and administrative angles are accounted for in Section VI, while a multidisciplinary sway assessment is introduced in Section VII. Finally, ends are attracted Section VIII.

\section{NEW USERNEEDS}

Over the most recent 50 years, life possibilities have prompted a rate increment of over $64 \mathrm{~s}$ and by 2020 there will be more 60-year-olds than kids under five. Italy positions in second situation on the planet for life span, after Japan [2] and the transitory wonder just mostly figures out how to hinder the elements of the dynamic maturing of the populace. This impressively influences the Health and Welfare framework: the steady increment of the old and the upgrades got in the future of a patient lead to a developing number of ceaseless patients after some time. The pace of ceaseless degenerative sicknesses increments with the age of the populace: considering individuals matured 55-59, 53\% experience the ill effects of an incessant malady, while for the more than 75 -year-old the rate arrives at $85.3 \%$. In Tuscany area, there are practically 1.5 million of constant patients, equivalent to $40.1 \%$ of the populace (Italian normal is $39.9 \%$ in 2017). Additionally, given the developing number of individuals who have more than one incessant sickness than the individuals who just have one, the most regular interminable condition is comorbidity (20.3\%). It is presently important to confront this issue with an alternate point of view, increasingly arranged to the general clinical intricacy of the individual, as opposed to the administration of individual pathologies. Contrasted with 2018, there are around 20000 increasingly ceaseless patients in Tuscany and this converts into a more noteworthy duty, particularly by nearby administrations, in the day by day the executives of the sickness [3]. Client focused wellbeing observing is usually utilized in human services and has increased across the board fame by encouraging wellbeing support and overseeing incessant conditions [4] and empowering individuals to add to their prosperity and wellbeing [5]. The WHO Global Observatory for E-Health has led three worldwide reviews on the worldwide spread of e-Health (in 2006, 2011 and 2016). It has become progressively certain that general wellbeing inclusion (UHC) can't be accomplished without eHealth support. Various nations have revealed in any event one mHealth activity (83\%).

\section{FUTURE WIRELESS HEALTHCARESERVICES}

The future social insurance framework should manage an expanding number of interminable patients and the absence of clinical assets to meet patients' consideration needs. This makes the reception of ICT essential for the anticipated wellbeing administrations. Concentrating on observing the wellbeing status of the patient experiencing different constant infections, the chance of a continuous estimation of some wellbeing pointers and the evaluation of the general status of the patient 
joined with the consequently sharing of the obtained clinical information among all the individuals from the consideration group is of central significance for customized the board of the patient wellbeing.

Accordingly, earlier recognizable proof of the potential wellbeing hazard and back to back arranging of proper activities, for example, adjustment and the executives of treatment or calendar of extra tests, will profit by the incorporation of creative innovative devices in wellbeing conventions and authoritative models. $6 \mathrm{G}$ can be viewed as the empowering innovation for the future remote social insurance administrations, which can't be cultivated effectively without the utilization of explicit ICT frameworks.

\section{Main Features of Future Services}

These days, general wellbeing divisions are confronting a huge advancement in every case increasingly centered around the combination of the heterogeneous characterized human services pathways from a calculated point of view as well as from a methodological stance through the upgrade of administrations and the sending of IoT (Internet of Things) arrangements and heterogeneous sensors [6].

Basically, yet not restricted to, in the essential social insurance the exertion is to put the patient at the focal point of the consideration pathways breaking down a particular infection as well as the general patient's wellbeing status and the whole condition around him/her.

Ideas, for example, "One Health" take the shape from the thought that individuals are remembered for an all around characterized biological system that unavoidably sway on individuals and their wellbeing status [7].

So as to appropriately handle the wellbeing challenges brought about by the general condition in every case progressively effective IoT administrations and sensors for clients and biological system examination are required. In such situation, $6 \mathrm{G}$ finds the opportunity to be the cornerstone and to be problematic in the human services area in view of its greater dependability and security.

\section{Future Services}

Future administrations will go towards a clinical science that will consider the person all in all framework and doesn't separate the clinical divisions. Actually, the treatment of an organ or a malady consistently includes way of life, including mental passionate and even natural parameters. Person is viewed overall framework comprising of body and mind, and the individual wellbeing doesn't only depend on the strength of the working of a solitary organ.

As indicated by this new all-encompassing worldview of the evaluation of the wellbeing status of an individual, the checking of all the wellbeing markers isn't simple. The selection of cutting edge sensors both inside and on the body for a consistently on and portable control is required. This requires an inescapable, dependable, protected and adaptable "connective tissue" which might be spoken to by $6 \mathrm{G}$ innovation.

Future e-wellbeing administrations dependent on the propelled checking of patients including fundamental sign, explicit wellbeing markers, physical action, mental perspectives, general condition (various information sources) can essentially improve the arrangement of customized social insurance by: I) facilitating the advancement of new and proficient exceptionally adjustable models of care; ii) empowering a precise and dynamic meaning of care needs; and iii) distinguishing the most appropriate wellbeing administration for every patient, helpfully supporting and directing the human services experts. 
The incorporation and connection of information (biometric parameters, physical movement, and adherence to treatment) through cutting edge Artificial Intelligence calculations will permit successful observing of the patient's wellbeing, giving a quick outline of the advancement of the taken estimations and a fast distinguishing proof of any oddities. This will empower the enactment of programmed alert for understanding counteraction/the executive's activities. Truth be told, acceptable consistence between some parametric/anthropometric checks and the recommended treatment is basic for improving or keeping stable patient wellbeing conditions, upgrading personal satisfaction, decreasing hospitalizations and in any event, for a progressively fitting utilization of analytic imaging and lab e-Health administrations may run from tele-observing for hazard counteraction to "keen medications" frameworks remotely actuated and from virtual tele-visits to cutting edge tele-restoration pathways. The utilization of bidirectional intuitive innovation and media communications additionally improves coordinated effort (continuous and huge correspondence) among all social insurance experts engaged with the patient consideration. The entirety of this in a roundabout way impacts on pharmaceuticals, diagnostics and pro costs and it helps decreasing grimness and death rates, hospitalization times and the expense of patient consideration. Besides, it likewise expands patients' self-administration, enabling and rousing them towards a progressively dynamic and powerful administration of their own wellbeing.

\section{G KEYFEATURES}

So as to present how $6 \mathrm{G}$ innovation can change the future remote medicinal services framework, a short review of the key highlights of $6 \mathrm{G}$ is given. While [8] centers around the meaning of $6 \mathrm{G}$ potential correspondence application situations and the related empowering interchanges innovations, a multidisciplinary $6 \mathrm{G}$ vision, including key drivers, inquire about necessities, difficulties and fundamental research questions is introduced in [9]. With the end goal of this paper it merits beginning from the five application situations bolstered by $6 \mathrm{G}$ correspondence advancements [8]:

- EMBB-Plus (Enhanced Mobile Broadband Plus). It is the replacement of eMBB of 5G and it should improve the presentation of versatile interchanges as far as obstruction, handover, information transmission, information preparing and interoperability yet additionally ensuring a significant level of security and protection.

- Big Com (Big Communications). It targets stretching out correspondence administrations to remote territories, by ensuring system inclusion and satisfactory nature of administration all over.

- SURLLC (Secure Ultra-Reliable Low-Latency Communications). It ought to give higher dependability than 5G URLLC and lower inactivity than 5G m MTC, including security angles.

- 3D-InteCom (Three-Dimensional Integrated Communications). It tends to 3D arrange investigation, arranging and streamlining, that considers the statures of system hubs (for example satellite, unmanned flying vehicle, submerged interchanges, full-dimensional MIMO models).

- UCDC (Unconventional Data Communications). It incorporates new correspondence models and standards that may run (yet are not restricted to) from holographic and material Internet to human-security interchanges. In outline, the principle necessities for $6 \mathrm{G}$ are the accompanying: rapid and high-limit correspondences, outrageous inclusion expansion, low force utilization, low dormancy, high solid interchanges, enormous availability and detecting and high security and protection. 
The expansion of the internet and of the selection of Big Data and AI innovations request improvements of security and protection. Then again, in spite of the fact that the improvement of the otherworldly effectiveness in $6 \mathrm{G}$ would not be for an enormous scope because of the accomplishments previously came to by $5 \mathrm{G}$ innovation, rapid and high-limit correspondences will be the reason for the arrangement of cutting edge virtual/enlarged client experience. Also, novel vitality proficiency arrangements, including low force utilization gadgets, are relied upon to be characterized in consistence with the green correspondence worldview. At long last, new system designs are required to adapt to the dynamicity of system topology and to guarantee high reasonableness and full customization dependent on cutting edge AI usage. 6G insight will be conveyed over the correspondence organize, including operational, natural and administration levels.

\section{G WIRELESS TECNOLOGY AS HEALTHCARESERVICES ENABLER}

The appropriation of frameworks described by huge network, ultra-low dormancy and high speeds, higher battery limit, information driven ideal models will influence the social insurance setting prompting the decentralization of human services model and to accuracy and customized medication in accordance with the dynamic idea of individual. Wearable and interconnected electronic gadgets, including bio incorporated ones (for example electronic skin, nanoscale smart frameworks, and so forth) are required for a continuous checking of wellbeing related parameters.

6G innovation is the key empowering agent for this social insurance framework change. It will bolster the arrangement of new administrations for the checking, assessment and treatment of the individual wellbeing status. The human body will be viewed as a system hub, including physical, mental and social parts in an all encompassing vision of the person.

The observing of people's wellbeing status targeting prosperity, counteraction, interminable multi-morbidities or incessant infection difficulties the board and treatment personalization requires the reconciliation of information originating from controlled randomized clinical preliminaries and information gathered by various wellsprings of data in a genuine consistently world. This present reality information may incorporate natural, physical action, mental, treatment, and day by day life propensities information accumulated by gadgets and versatile applications, ecological sensors, open and private social insurance suppliers, and so forth. The corresponding highlights of these information may give new bits of knowledge into sickness designs, bolster medicinal services choice and improve the adequacy of potential wellbeing mediations.

Propelled frameworks for information investigation dependent on computerized reasoning and AI calculations permit a multi-reason understanding of an enormous measure of these heterogeneous procured information. The relationship principally among clinical, mental, way of life and ecological information will assist with forestalling and improve the people's wellbeing status, moving towards an all-encompassing vision of care. Concentrating on the correspondence frameworks, a few instances of how 6G will empower these administrations are given.

\section{In-body, on-body, off-body Communications}

Focusing on tele-checking the wellbeing status of people through the reception of ICT frameworks, the programmed securing of information from person's (Body-Layer) speaks to a key component. The Body-Layer remembers for body, onbody and off-body correspondences that empower the social event of various data through in-body and on-body sensors and the transmission of information out of the person's body. 
In-body sensors are spoken to by nano-gadgets and additionally embeds, or particles going about as natural correspondences frameworks, while on-body sensors are wearable gadgets for bio-parameters estimations (e.g.: circulatory strain, beat oximeter, ECG sensors) obtaining. Then again, off-body correspondences permit the client straightforward and constant transmission of information to edge gadgets and to the cloud for short-and long haul examination, individually.

\section{Intelligent Nanoscale Inner Body Communications}

The interconnection of nanoscale gadgets dependent on nanomaterials (for example graphene) prompts the worldview of Internet of NanoThings (IoNT). Be that as it may, the counterfeit idea of IoNT gadgets and its potential undesirable impacts on wellbeing (for explicit wellbeing related applications) makes scientists center around a novel methodology: the Internet of Bio-Nano Things (IoBNT) [10]. The correspondence and system designing ideas are moved in the biochemical area, where natural cells inside the human body (Bio-Nano Things) empower intra-body detecting and incitation. Wellbeing information are gathered and transmitted to the wellbeing supplier and execution required activities are remotely enacted (for example discharge drugs) in view of the assembled data.

The meaning of new system designs and conventions for interconnecting heterogeneous bio-nano systems and for interfacing them with the encompassing digital condition are required. In this specific situation, Molecular Communications (MC) frameworks are fundamental to permit trade of data among cells. Considering concoction forms, data signals are balanced into substance qualities (for example particle focus, type and vitality state) and traded among cells. Distinctive cell flagging pathways are characterized dependent on the separation between the source $(\mathrm{S})$ and the goal (D). These are: intracrine ( $\mathrm{S}$ and $\mathrm{D}$ inside a similar cell), juxtracrine ( $\mathrm{S}$ and $\mathrm{D}$ are cells in contact with one another), paracrine ( $\mathrm{S}$ and $\mathrm{D}$ close however not in contact), or endocrine ( $\mathrm{S}$ and $\mathrm{D}$ removed).

6G innovation assumes a key job in this specific circumstance and will empower the interconnection of IoBNT and IoNT to the IoT, giving an intend to the acknowledgment of the vision of "everything associated". In any case, new security instruments are required to adapt to the likelihood to get to the human body with malignant expectation (for example bio-digital fear based oppression), and furthermore confinement and following procedures inside the IoBNT are required for sickness area distinguishing proof, drugs nearby discharge, and so forth.

\section{Human Bond Communications}

- Vivid and instinctive encounters not restricted to the advanced transmission of aural and optical data can be accomplished through the mix of the data originating from the other three tangible highlights: olfactory, gustatory and material. Human Bond Communication (HBC) speaks to a novel methodology dependent on the recognition and transmission of data utilizing each of the five human detects (sight, smell, sound, contact, and taste). It is characterized as an all-encompassing way to deal with depict and transmit the highlights of a subject in the manner people see it [11] [12].

- In the social insurance settings, completely checking and recreating the faculties to a remote area prompts a comprehensive correspondence mean of patients' wellbeing status, helping human services specialists in settling on significant and ideal information driven choices for their patients.

- In the following future, the transduction of "musings" or impressions of an individual (for example electrical trades of the mind) and their transmission to another individual may become reality. In any case, to get this going an "adaptable connective texture" of media communications that begins from inside the body and arrives at the 
last client through heterogeneous sections (center, cloud, and so on.) is required. 6G can be viewed as an innovative "texture" that may empower a drawing in client experience trough the impression of the more sensorial passionate data of the human body. This encourages the arrangement of cutting edge, customized and inescapable administrations extending from patients' checking, finding, help and treatment to the expansion of the discerning capacities of a person, tending to likewise mental and social perspectives.

\section{Visible Light Communication}

Obvious Light Communication (VLC) is an optical remote correspondence innovation which depends on the utilization of regulated noticeable light waves as a vehicle of data transmission. It is described by high information rate, intrinsic secure transmission, low vitality utilization and no radio recurrence radiation (prompting less human wellbeing risky impacts). Because of these points of interest, VLC is a legitimate contender for the arrangement of future remote medicinal services benefits in indoor settings. It tends to be incorporated with the current remote advances [13] and can be utilized both for social affair data from the individual body [14] and for "thick" correspondences among clients likewise misusing the lighting framework, which effectively give a downlink channel.

\section{CHALLENGES}

\section{Privacy and Security}

Social affair data from in-and on-body sensors and transmitting the gathered information towards a cloud clinical Center for additional examination request new security arrangements.

Specifically, because of the restricted assets of the body sensors as far as vitality utilization and calculation capacities, accomplishing secure correspondence inside and on the body despite everything speaks to an open research zone. The fundamental difficulties emerge from: I) the restricted assets (power, calculation, stockpiling limit and so forth.) of nano devices, which make unfeasible the appropriation of on-request key conveyance strategies and troublesome the usage of pre-sent key methods; and ii) the need of giving versatile system in a nano-space [15]. Accordingly, unique confirmation vitality productive calculations and shorter key length cryptographic systems along with new correspondence and security structures are required. A few examinations have been completed in the structure of atomic interchanges both for a fundamental assessment of the mystery limit in a dispersion based MC framework [16] and for proposing new wellsprings of encryption dependent on natural particles (for example DNA and RNA). Be that as it may, particularly in nano-scale settings, further examination is as yet expected to adapt to particles unconstrained response and wild situations.

\section{Technological Aspects}

The joining of detecting, high goals imaging and high exact situating on versatility, imagined by the 6G innovation, will open new administrations, specifically in the wellbeing area. The expanding enthusiasm of logical research to in-body nano-gadgets (both wet and dry), including biochemical interchanges, will drive the future importance of body as a major aspect of the system. Association and collaborations between natural cells and electrical nanodevices isn't any longer incomprehensible. The likelihood to impart both utilizing electromagnetic waves and biochemical responses/particles dispersion can make the human body a correspondence situation, as the fiber or the ether. The Internet of Bio-Nano-Things worldview incorporates the two inserts, nano-scale counterfeit gadgets just as natural combined particles. All these are components of a system and can convey through explicit systems administration gadgets. Cooperation between in-body and on-body hubs will permit to recuperate significant data from extremely inside the body and course it to the cloud. All 
these various types of hubs, from exceptionally minuscule to large scale, have altogether different prerequisites to impart data. $6 \mathrm{G}$ innovation will be the texture that can make all these various components part of a solitary system, permitting a persistent, reliable and secure dissemination of data.

The investigations about people incorporate, consistently, the mystic/feeling circle. Mental angles are critical for the strength of an individual. The data recovered by in-and on-body sensors can be utilized to deduce not just the wellbeing from a natural perspective, yet additionally from an enthusiastic one. Information originating from consistent checking of human body during the regular daily existence can be joined/related to obtain an all out vision of the wellbeing status of the individual, and $6 \mathrm{G}$ innovation can manage the cost of the network for this, on account of its natural unavoidable and versatile attributes on portability.

In June 2019, the Panel published the report "The Age of Digital Interdependence" and with it a series of recommendations to improve digital cooperation. While no single vision emerged, there was broad agreement that "governments, the private sector and civil society will need to find new ways to work together to steer an effective path between extremes of over-regulation and complete laissez- faire". According to the Report a multi-stakeholder "systems" approach for digital cooperation and regulation that is adaptive, agile, inclusive and fit for purpose for the fast-changing digital age is necessary. A concrete recommendation desirable in a $6 \mathrm{G}$ scenario, deals with, where possible, to test new regulatory approaches on a small scale before being rolled out widely - through, for example, trial periods, or pilot zones, where experimentation with different levels of regulation can be carried out in a controlled environment to test their impact.

\section{MULTI-DISCIPLINARY IMPACTEVALUATION}

Obtaining these goals requires facing important challenges form the technological point. of view Gathering data from very different devices, from nano to macro, and communicating in heterogeneous type of channels, from chemical to radio or free-space optical, will face important challenges,as1) propagation in different environments, from inside the human body to atmosphere or fiber; 2) different devices capability; 3) high accurate positioning; 4) ultra-fast computing and delivery of information to destination; 5) harvest energy in every environment and wireless energy transfer; 6) resource management and networking of heterogeneous data from heterogeneous sources; 7) interference management and mitigation; 8) ultrafast machinelearningandartificialintelligence;9) access schemes formassivewirelessnetworks;10) cyber security.

\section{Ethical Aspects}

6G can enable, on a massive scale, the use of multiple and heterogeneous data-driven decision-making system that can drive the transformation of preventive and healthcare pathways towards real needs of individuals and society.

In order to drive such process ethically, it is fundamental to involve citizens in a participative process that requires the increase of health literacy, and the empowerment of citizens as data owner.

In parallel, the digital divide poses the challenge of equity in the access to healthcare. An increasingly digitised world risks leaving marginalized people behind.

The increase in interconnectivity rein force our underlying interdependence, thus Digital Cooperation across domains and across borders is critical to realize the transformational potential of digital technologies, while safeguarding 
against risks and unintended consequences [17]. Therefore, establishing common regulatory frame work is of paramount importance in order to face the challenges of the forth coming $6 \mathrm{Gera}$.

\section{Regulatory Aspects}

In July 2018 the Secretary-General of the United Nations appointed the High-level Panel on Digital Cooperation. The Panel mission is to identify good practices and suggest modalities for working cooperatively across sectors, disciplines and borders to address the social, ethical, legal and economic impact of digital technologies, in order to maximise their benefits and minimise their harm[17].

$6 \mathrm{G}$ compared to $5 \mathrm{G}$ get the chance to make a concrete step forward in terms of functionalities and quality of the experience shifting the focus on services (and not more on the users), enforcing a service's reliability, deploying VAR (Virtual Augmented Reality) on a massive scale and concretely rooting Smart City components. This causes massive impact even in the healthcare sector. The following subsections try to highlight the potential impacts for three actors of the health domain, namely: a) patients, b) healthcare professionals, and c) Society.

\section{Patient's Point of View}

$6 \mathrm{G}$ finds the opportunity to create a bigger effect on the nature of social insurance got by patients and to fuel huge changes in the manner by which human services is given and could be a considerable empowering agent of another kind of "customized medicinal services" approach.

The a lot more noteworthy capacity to persistently accumulate tolerant explicit information and the capacity to process, break down and rapidly return prepared data and prescribed blueprints to the patient will give patients more noteworthy capacity to oversee conditions on their own contemplating wellbeing related information as well as natural data. The personalization of medicinal services likewise implies that doctors and other social insurance suppliers will have the option to make "without a moment to spare" judgments and tailor cures all the more near a specific patient's very own needs because of the higher and increasingly dependable measure of information gathered from an enlarged assortment of sources (AI advancements, IoT arrangements, HBC sensors, in-body, on-body off-body correspondence sensors and so forth.). This could open the entryways for a decrease of the quantity of re-hospitalizations and gives the chance to improve patients' cash reserve funds and mental pressure.

Also, 6G will quality the patients ceaseless observing in this way improving the viability of preventive consideration. Actually, through $6 \mathrm{G}$, nonstop checking will uphold the improvement of new extra information streams therefore implementing prescient examination and keen [18].

\section{Healthcare Professionals' Point of View}

6G technology may change the future healthcare system enabling the achievement of new goals from the healthcare professional's point of view. Some of them are:

- The increase of the quality of the primary and homecare system through real-time monitoring and information sharing of patient's condition and pharmacological efficacy for a constantly updated and revised care path.

- The improvement of the quality of life through proactivity by maintaining the patient's residual functional capacities and preventing the deterioration and disabilities of patients' health. 
- The rationalization of health costs through the reduction of improper access to hospital and health services, the reduction of expenses for non-targeted, specialized and diagnostic drug therapies, etc.

- The economic and financial sustainability of a system in which chronic and highly personalized patient care is mainly carried out at the patient's home.

- The dynamic adaptation of health policies to the ongoing evolution of the population needs by orienting resources towards the implementation of services based on the changing population health.

\section{Society}

From the general public perspective, the predictable impacts from the $6 \mathrm{G}$ 's sending are numerous. In reality, $6 \mathrm{G}$ sending has the possibility to totally adjust the social insurance framework establishing the framework to a progressively adaptable and customized framework in which assets are appropriately dispensed by a "win-win" approach planned for decreasing the misallocation of assets (human and fiscal assets) and their waste. Truth be told, because of the improved personalization of social insurance, $6 \mathrm{G}$ can possibly affect on open human services framework and private clients cost investment funds and profitability enhancements: this is the situation in the event that we consider the predominant administration of long haul and interminable conditions. Furthermore, 6G could strengthen the locus of care move from medical clinics to homes and other lower-cost settings along these lines offering another wellspring of cost-reserve funds [19]. Moreover, 6G, on account of its troublesome behavior, could support the difference in the business biological system by opening the market to new partners like smaller scale administrators and asset intermediaries, empowering numerous new plans of action yet additionally reviving the old ones.

\section{CONCLUSIONS}

Wellbeing is one of the vertical of 6G. 6G innovation will assume a key job in giving productive and financial patient remote administrations and will bolster human services experts in their day by day exercises. They will ease and empower quiet consideration at home, adapting both to the developing number of patients and the absence of social insurance experts' assets. The $6 \mathrm{G}$ framework is here broke down as a potential answer for meet the necessities of the remote social insurance of things to come. The $6 \mathrm{G}$ innovations guarantees are here subtleties, just as the difficulties that must be looked to offer the new types of assistance in the social insurance arrangement of 2030.

\section{REFERENCES}

1. European Commission, Orientations towards the primary Strategic Plan for Horizon Europe, December 2019

2. World Health Assembly. Goals WHA51.7. Wellbeing for all arrangement for the twenty-first century. Geneva: World Health Organization, 1998. 2019, "Welfare e salute in Toscana". http://www.regione.toscana.it/reports/10180/13809783/Welfare+e +Salute+2019+vol+1/80c78777-e16e-4f22-ab43-bc9003bb095c last get to January 152019.

3. Sarkar U., Gourley G. I., Lyles C. R., Tieu L., Clarity C.,NewmarkL.,Singh K., Bates D.W. , 2016, "Convenience of monetarily accessible versatile applications for differing patients", Journal of General Internal Medicine, 31(12), 1417-1426.

4. Mahalakshmi, B. Naga, and V. Vara Prasad. "Study on Mechanical Behaviour of Carbon Fiber Reinforced Epoxy Composites." International Journal of mechanical and production engineering research and development (2013). 
5. Birkhoff S., Moriarty H., 2016, "Intercessions utilizing cell phone wellbeing applications across different populaces: An integrative audit of the writing", Journal of Informatics Nursing,1(1), 13-24.

6. WHO, "Coordinated consideration models: an outline", Health Services Delivery Program Division of Health Systems and Public Health, 2016.

7. World Bank, "One Health; Operational structure for reinforcing human, creature, and natural general wellbeing frameworks at their interface, World Bank Group, 2018.

8. Selvam, Rajiv, and Surjith Jacob. "Experimental Investigation and Analysis of Smaw Processed Carbon Steel Pipes." International Journal of Mechanical and Production Engineering Research and Development 8.5 (2018): 29-40.

9. Dang, S., Amin, O., Shihada, B. et al. What ought to 6 G be?. Nat Electron 3, 20-29 (2020). https://doi.org/10.1038/s41928019-0355-6

10. Aazhang, Behnaam and Ahokangas, Petri and Alves, Hirley and Alouini, Mohamed-Slim and Beek, Jaap and Benn, Howard and Bennis, Mehdi and Belfiore, Jean and Strinati, Emilio and Chen, Fan and Chang, Kapseok and Clazzer, Federico and Dizit, Sudhir and DongSeung, Kwon and Giordiani, Marco and Haselmayr, Werner and Haapola, Jussi and Hardouin, Eric and Harjula, Erkki and Zhu, Peiying. (2019). Key drivers and research difficulties for 6 G pervasive remote knowledge (white paper).

11. Slamet, Riskiono. "Fabrication and Characterization of Eriochrom Cyanine R Entrapped Sol-Gel Membrane for Determination of Aluminium (III). "International Journal of Chemical \& Petrochemical Technology (IJCPT) 3. 3, Aug 2013, 1-8

12. I. F. Akyildiz, M. Pierobon, S. Balasubramaniam and Y. Koucheryavy, "The web of Bio-Nano things," in IEEE Communications Magazine, vol. 53, no. 3, pp. 32-40, March 2015. doi: 10.1109/MCOM.2015.7060516T.

13. E. Del Re, S. Morosi, L. Mucchi, L.S. Ronga, S. Jayousi, "Future Wireless Systems for Human Bond Communications", Wireless Personal Communications - Springer (2016) 88:39-52, DOI 10.1007/s11277-016-3240-x

14. Phuntulee, Siriporn, et al. "The Pattern of Health Management with Self-Reliance Based on Mpi People Local Wisdom, Phrae Province, Thailand." International Journal of Educational Science and Research (IJESR) 6.3 (2016).

15. Iftikhar, H. A. Khattak, Z. Ameer, M. A. Shah, F. F. Qureshi and M. Z. Shakir, "Human Bond Communications: Architectures, Challenges, and Possibilities," in IEEE Communications Magazine, vol. 57, no. 2, pp. 19-25, February 2019. doi: 10.1109/MCOM.2018.1800531

16. Ahmed, I., Karvonen, H., Kumpuniemi, T. et al. Remote Communications for the Hospital of the Future: Requirements, Challenges and Solutions. Int J Wireless Inf Networks (2019) doi:10.1007/s10776-019-00468-1

17. Mahalakshmi, K. also, M, Sivaram and Kumari, K. Shantha and Yuvaraj, D and Keerthika, R., Healthcare Visible Light Communication (2018). Universal Journal of Pure and Applied Mathematics, Volume 118 No. 11 2018, 345-348. Accessible at SSRN: https://ssrn.com/abstract $=3352115$

18. Mucchi, S. Jayousi, A. Martinelli, S. Caputo and P. Marcocci, "An Overview of Security Threats, Solutions and Challenges in WBANs for Healthcare," 2019 thirteenth International Symposium on Medical Information and Communication Technology (ISMICT), Oslo, Norway, 2019, pp. 1-6. doi: 10.1109/ISMICT.2019.8743798

19. L. Mucchi, A. Martinelli, S. Jayousi, S. Caputo and M. Pierobon, "Mystery Capacity and Secure Distance for Diffusion-Based Molecular Communication Systems," in IEEE Access, vol. 7, pp. 110687-110697, 2019. doi: 10.1109/ACCESS.2019.2932567

20. United Nations, The time of advanced association, Secretary-General's High-level Panel on Digital Cooperation Report, 2019. 
21. Teece D., "5G portable: Impact on the Healthcare segment", Tusher Center for Intellectual Capital Institute for Business Innovation Haas School of Business U.C. Berkeley, October 2017.

22. Seppo Yrjölä, "Decentralized 6G Business Models", meeting paper, 04/2019 